\title{
On Optimal Periodic Control and Nested Optimization Problems ${ }^{1}$
}

\author{
F. COLONIUS ${ }^{2}$
}

\begin{abstract}
A minimization problem for a functional on a convex subset $C$ of a normed linear space is considered. Under certain hypotheses, optimality in a certain subset of $C$ implies the validity of first-order necessary optimality conditions for the problem in $C$. The result is applied to a problem in optimal periodic control of neutral functional differential equations.
\end{abstract}

Key Words. Optimal periodic control, first-order optimality conditions, neutral functional differential equations.

\section{Introduction}

This note is motivated by optimal periodic control problems. Here, a fundamental problem is to discern optimal steady-state solutions which are merely optimal among steady states from those which are also optimal among periodic solutions (see e.g. Ref. 1 and Section 3 below). For systems governed by ordinary differential equations, it is well known that first-order necessary optimality conditions for optimality in the (restricted) class of steady states coincide with the corresponding conditions for optimality in the larger class of periodic solutions. This excited interest in higher-order optimality conditions (see, e.g., Refs. 1-3).

In Ref. 4, it was shown that a similar result holds for optimal periodic control of retarded functional differential equations by deriving the concrete

\footnotetext{
${ }^{1}$ This work was partially supported by a grant from Deutsche Forschungsgemeinschaft and by AFOSR under Grant No. AFOSR-84-0398.

${ }^{2}$ Visiting Assistant Professor, Lefschetz Center for Dynamical Systems, Division of Applied Mathematics, Brown University, Providence, Rhode Island. Present address: Institut für Dynamische Systeme, Universität Bremen, D-28 Bremen 33, West Germany.
} 
form of the optimality conditions. This, however, required a lot more effort and induced the question of which particular features of the underlying optimization problem(s) are responsible for this phenomenon.

Section 2 gives a simple characterization of two nested optimization problems leading to identical first-order optimality conditions. Section 3, illustrates that this abstract result captures in fact the relevant features of optimal periodic control problems. Here, we deal with neutral functional differential equations (including, in particular, the standard optimal periodic control problem for ordinary differential equations). Functional differential equations are of interest in this context, since, in control problems for chemical reactions, delays occur frequently (e.g., due to recycle loops). These problems, besides flight performance optimization, are a major field of applications for optimal periodic control.

\section{Nested Optimization Problems}

Consider the following optimization problem:

(P) minimize $g(y)$, s.t. $y \in C \subset Y$;

here, $\mathcal{O}$ is an open subset of the normed linear space $Y, g: \mathcal{O} \rightarrow \mathbb{R}$ has a Gateaux derivative $g^{\prime}\left(y_{0}, y\right)$ at $y_{0} \in C$ in direction $y \in Y$, and $C$ is a closed and convex subset of $Y$. A first-order necessary optimality condition for a local minimum $y^{0} \in \mathcal{O}$ has the form

$$
g^{\prime}\left(y^{0}, y-y^{0}\right) \geq 0
$$

for all $y \in C$. As is well known, there are many problems where this condition is not only satisfied by local minima, but by other points $y^{0}$, too. Most frequently, this occurs when $g^{\prime}\left(y^{0}, \cdot\right)=0$. In such a case, one has to seek recourse to either higher-order necessary optimality conditions or to sufficient optimality conditions (see Ref. 5).

Below, we describe a situation, where points $y^{0}$ which are only optimal with respect to a certain subset $\tilde{C}$ of $C$ satisfy condition (1) not only for elements $y$ in $\tilde{C}$, but for all elements $y$ in $C$.

Thus, we consider, in addition to the problem (P) formulated above, the following minimization problem, sitting inside $(\mathrm{P})$ :

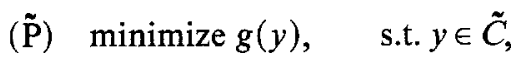
where $\tilde{C}$ is a subset of $C$. We have the following result.

Proposition 2.1. Let $y^{0} \in \tilde{C}$ satisfy the first-order optimality condition for $(\tilde{P})$; i.e., suppose that (1) holds for all $y \in \tilde{C}$. Assume that there exists 
a linear projection $\mathscr{P}: Y \rightarrow \tilde{Y}$, where $\tilde{Y} \subset Y$ is a linear subspace containing $\tilde{C}$ such that

(i) $g^{\prime}\left(y^{0}, y\right)=g^{\prime}\left(y^{0}, \mathscr{P}_{y}\right), \quad$ for all $y \in Y$;

(ii) $\mathscr{P} C \subset \tilde{C}$.

Then, $y^{0}$ satisfies the first-order optimality condition for a local minimum of problem (P), i.e., (1) holds for all $y \in C$.

Proof. By assumption,

$$
g^{\prime}\left(y^{0}, \tilde{y}-y^{0}\right) \geq 0, \quad \text { for all } \tilde{y} \in \tilde{C} \text {. }
$$

Now, let $y \in C$. By assumptions (i) and (ii), the inequality (2) implies

$$
g^{\prime}\left(y^{0}, y-y^{0}\right)=g^{\prime}\left(y^{0}, \mathscr{P}\left(y-y^{0}\right)\right)=g^{\prime}\left(y^{0}, \mathscr{P} y-y^{0}\right) \geq 0,
$$

since $\mathscr{P}$ is linear.

Remark 2.1. Suppose that $\tilde{C}$ is closed and convex. Then, every locally optimal solution $y^{0}$ of $(\tilde{\mathrm{P}})$ satisfies (1) for all $y \in \tilde{C}$.

Remark 2.2. Suppose that $Y$ is a Hilbert space, $\tilde{Y}$ is a closed linear subspace of $Y$, the set $\tilde{C}$ is given by $\tilde{C}:=C \cap \tilde{Y}$, and $g$ is Fréchet differentiable at $y^{0} \in \tilde{C}$. Thus, $g^{\prime}\left(y^{0}\right)$ is a continuous linear functional on $Y$. Hence, by the Riesz representation theorem, $g^{\prime}\left(y^{0}\right)$ can be identified with an element of $Y$. Take $\mathscr{P}$ as the orthogonal projection of $Y$ onto $\tilde{Y}$. Then, conditions (i) and (ii) above are equivalent to

$$
g^{\prime}\left(y^{0}\right) \in \tilde{Y} \text { and } \mathscr{P} C \subset C \text {. }
$$

Remark 2.3. Impose in problems $(\mathrm{P})$ and $(\tilde{\mathrm{P}})$ the additional constraint

$$
F(y)=0 \text {, }
$$

where $F: Y \rightarrow Z$ is continuously Fréchet differentiable at $z^{0}$ and $Y, Z$ are Banach spaces. Under an appropriate regularity condition (Ref.5), necessary optimality conditions for the modified problems $(P)$ and $(\tilde{\mathbf{P}})$ have the following form: There exist $\lambda_{0} \in \mathbb{R}_{+}$and a continuous linear functional $z^{*}$ on $Z$ (i.e., $z \in Z^{*}$ ), with $\left(\lambda_{0}, z^{*}\right) \neq(0,0)$, such that

$$
\lambda_{0} g^{\prime}\left(z^{0}\right)\left(y-y^{0}\right)+z^{*}\left(F^{\prime}\left(y^{0}\right)\left(y-y^{0}\right)\right) \geq 0,
$$

for all $y \in C$ [resp., all $y \in \tilde{C}]$. Suppose that, in Proposition 2.1, the condition (1) is replaced by (3) for some pair $\left(\lambda_{0}, z^{*}\right) \in \mathbb{R} \times Z^{*}$ and, additionally, the following condition is satisfied:

$$
\text { (iii) } z^{*}\left(F^{\prime}\left(y^{0}\right) y\right)=z^{*}\left(F^{\prime}\left(y^{0}\right) \mathscr{P} y\right), \quad \text { for all } y \in Y \text {. }
$$

Then, the assertion of the proposition remains valid. 


\section{Optimal Periodic Control for Neutral Functional Differential Equations}

Consider a neutral functional differential equation of the form

$$
(d / d t) D x_{t}=f\left(x_{t}, u(t)\right), \quad t>0,
$$

where $r>0$ denotes the length of the delay, $x_{t}(s):=x(t+s) \in \mathbb{R}^{n}, s \in[-r, 0]$, $u(t) \in \mathbb{R}^{m}$, and $f: C\left(-r, 0 ; \mathbb{R}^{n}\right) \times \mathbb{R}^{m} \rightarrow \mathbb{R}^{n}$. The map $D: C\left(-r, 0 ; \mathbb{R}^{n}\right) \rightarrow \mathbb{R}^{n}$ is supposed to be linear, continuous, and represented by a Riemann-Stieltjes integral

$$
D \phi=\phi(0)-\int_{-r}^{0}[d \mu(\theta)] \phi(\theta),
$$

where $\mu(\theta)$ is a $n \times n$ matrix of functions $\mu_{i j}(\theta)$, which have bounded variation $\operatorname{var}_{[-r, \theta]} \mu_{i j}$ and are left continuous on $(-r, 0)$ with $\mu_{i j}(0)=0$. Furthermore, we assume that

$$
\operatorname{var}_{[-s, 0]} \mu_{i j} \rightarrow 0, \quad s \rightarrow 0 .
$$

If $\mu=0$, then we obtain functional differential equations of retarded type,

$$
\dot{x}(t)=f\left(x_{t}, u(t)\right),
$$

which reduce to ordinary differential equations for $r=0$. If $\mu$ is piecewise constant, e.g.,

$$
\begin{aligned}
& \mu(-r)=A_{-1} \in \mathbb{R}^{n \times n}, \\
& \mu(t)=0, \quad t \in(-r, 0],
\end{aligned}
$$

and

$$
f(\phi, u):=A_{0} \phi(0)+A_{1} \phi(-r),
$$

we get a neutral functional differential equation of the form

$$
\dot{x}(t)=A_{0} x(t)+A_{1} x(t-r)+A_{-1} \dot{x}(t-r) .
$$

For the fundamental theory of these equations, we refer to Ref. 6 .

We assume here that, for every initial function $x_{0}=\phi \in C\left(-r, 0 ; \mathbb{R}^{n}\right)$ and every admissible control $u$ in

$$
u_{\mathrm{ad}}=\left\{u \in L_{\infty}\left(0, \tau, \mathbb{R}^{m}\right): u(t) \in \Omega \text { a.e. }\right\},
$$

where $\Omega \subset \mathbb{R}^{m}$ is closed and convex, there exists a unique absolutely continuous solution $x$ of (4). 
A corresponding optimal periodic control problem (OPC) has the following form:

$$
\operatorname{minimize}(1 / \tau) \int_{0}^{\tau} h(x(t), u(t)) d t
$$

subject to (4) and

$$
\begin{aligned}
& x_{0}=x_{\tau}, \\
& u \in U_{\mathrm{ad}} ;
\end{aligned}
$$

here, $h: \mathbb{R}^{n} \times \mathbb{R}^{m} \rightarrow \mathbb{R}$ is subject to the usual smoothness and boundedness assumptions. Clearly, (OPC) may be formulated as a minimization problem over $(\phi, u) \in C\left(-r, 0 ; \mathbb{R}^{n}\right) \times L_{\infty}\left(0, \tau ; \mathbb{R}^{m}\right)$, under the constraints $u \in \mathscr{U}_{\mathrm{ad}}$ and $x_{\tau}=\phi$, where $x$ is the unique solution of (4) with initial condition $x_{0}=\phi$ (see Ref. 4 for the retarded case). form:

The pertinent optimal steady-state problem (OSS) has the following

minimize $h(x, u)$, over $x \in \mathbb{R}^{n}, u \in \mathbb{R}^{m}$,

$$
\begin{array}{ll}
\text { s.t. } & 0=f(\bar{x}, u), \\
& u \in \Omega,
\end{array}
$$

where, for $x \in \mathbb{R}^{n}$, the function $\bar{x} \in C\left(-r, 0 ; \mathbb{R}^{n}\right)$ is the constant $\bar{x}(s) \equiv x$.

Suppose that $\left(x^{0}, u^{0}\right) \in \mathbb{R}^{n} \times \mathbb{R}^{m}$ is a locally optimal solution of (OSS). We assume that Eq. (4) can be linearized around $\left(\bar{x}^{0}, \tilde{u}^{0}\right)$, yielding

$$
(d / d t) D x_{t}=f_{x}\left(\bar{x}^{0}, u^{0}\right) x_{t}+f_{u}\left(\bar{x}^{0}, u\right) u(t), \quad t>0 .
$$

Suppose that (9) has, for every $\tau$-periodic control, a unique $\tau$-periodic solution $x$, that there is a neighborhood $V \times \mathcal{U}$ of $\left(\bar{x}^{0}, \bar{u}^{0}\right)$ in $C\left(0, \tau ; \mathbb{R}^{n}\right) \times$ $L_{\infty}\left(0, \tau ; \mathbb{R}^{m}\right)$ such that, for every $u \in U$, there exists a unique $\tau$-periodic solution $x$ of (4) in $V$, and $u \mapsto x(u)$ has a continuous Fréchet derivative. Now, we can fit these problems into the general framework of Section 2.

Define, for $u \in \mathscr{U}$,

$$
g(u)=(1 / \tau) \int_{0}^{\tau} h(x(t), u(t)) d t
$$

where $x$ is the unique $\tau$-periodic solution of (3) in $V$ corresponding to $u$, and let

$$
\begin{aligned}
& Y:=L_{\infty}\left(0, \tau ; \mathbb{R}^{m}\right), \quad \tilde{Y}:=\{u \in Y: u(t)=\text { constant a.e. }\}, \\
& C:=U_{\mathrm{ad}}, \quad \tilde{C}:=C \cap \tilde{Y} .
\end{aligned}
$$


We identify $\tilde{Y}$ with $\mathbb{R}^{m}$. The assumptions above guarantee that, for every $u \in \mathbb{R}^{m}$, there exists a unique constant solution $x$ of (9). Hence, by the implicit function theorem, there exists a neighbourhood of $\left(x^{0}, u^{0}\right)$ in $\mathbb{R}^{n} \times \mathbb{R}^{m}$, such that (7) has a unique solution $x$ for every $u$ with $(x, u)$ in this neighbourhood. This $x$ will also solve (4). Hence, we can consider (OSS) locally around $u^{0} \in \mathbb{R}^{m}$ as the problem to minimize $g(u)$ over $u \in \tilde{C}$.

Define the map $\mathscr{P}: Y \rightarrow \tilde{Y}$ by

$$
\mathscr{P} u:=(1 / \tau) \int_{0}^{\tau} u(t) d t
$$

Then, condition (ii) of Proposition 2.1 is satisfied since $\Omega$ is convex. Furthermore, the periodic solution $x^{0}$ of (4) corresponding to $u^{0}$ is constant, and the derivative $g^{\prime}\left(u^{0}\right) u$ is given by

$$
g^{\prime}\left(u^{0}\right) u=(1 / \tau) \int_{0}^{\tau}\left[h_{x}\left(x^{0}, u^{0}\right) x(t)+h_{u}\left(x^{0}, u^{0}\right) u(t)\right] d t
$$

where $x$ is the unique $\tau$-periodic solution of (9) corresponding to $u$. Since $x^{0}$ and $u^{0}$ are constant, we get

$$
g^{\prime}\left(u^{0}\right) u=h_{x}\left(x^{0}, u^{0}\right)(1 / \tau) \int_{0}^{\tau} x(t) d t+h_{u}\left(x^{0}, u^{0}\right)(1 / \tau) \int_{0}^{\tau} u(t) d t
$$

Clearly, $(1 / \tau) \int_{0}^{\tau} x(t) d t$ is the $\tau$-periodic solution of (9) corresponding to

$$
\mathscr{P} u=(1 / \tau) \int_{0}^{\tau} u(t) d t
$$

Hence,

$$
g^{\prime}\left(u^{0}\right) u=g^{\prime}\left(u^{0}\right) \mathscr{P} u
$$

and we have verified assumption (i) in Proposition 2.1. This proves the following result.

Proposition 3.1. Assume that the assumptions imposed above on (OPC) and (OSS) are satisfied, and let $\left(x^{0}, u^{0}\right) \in \mathbb{R}^{n} \times \mathbb{R}^{m}$ be a local optimal solution of (OSS). Then, $\left(x^{0}, u^{0}\right)$ satisfies the first-order necessary optimality conditions for a solution of (OPC).

The analysis above was based on the assumption that, for every $\tau$ periodic control $u \in U$, there exists a unique $\tau$-periodic solution $x$ of (9). For ordinary differential equations, this rules out the case that the matrix $f_{x}\left(x^{0}, u^{0}\right)$ is singular. This strong assumption was introduced in order to reduce the optimal periodic control problem (OPC) to an optimization problem over $u$, without implicit constraints, so that Proposition 2.1 applies. We indicate briefly how, for ordinary differential equations.

$$
\dot{x}(t)=f(x(t), u(t)),
$$


one can omit this undesirable assumption. Consider (OPC) as a minimization problem over pairs $(x, u) \in Y:=C\left(0, \tau ; \mathbb{R}^{n}\right) \times L_{\infty}\left(0, \tau ; \mathbb{R}^{m}\right)$ under the constraints $u \in U_{\mathrm{ad}}$ and

$$
\begin{aligned}
& x(t)-x(0)-\int_{0}^{t} f(x(s), u(s)) d s=0, \quad t \in[0, \tau], \\
& x(0)-x(\tau)=0 .
\end{aligned}
$$

The constraints (12)-(13) can be rewritten as

$$
\begin{aligned}
F(x, u) & =\left[\left(x(t)-x(0)-\int_{0}^{t} f(x(s), u(s)) d s, t \in[0, \tau]\right), \quad(x(0)-x(\tau))\right] \\
& =[0,0],
\end{aligned}
$$

when $F: Y \rightarrow Z:=C\left(0, \tau ; \mathbb{R}^{n}\right) \times \mathbb{R}^{n}$.

Let

$$
\begin{aligned}
& \tilde{Y}:=\{(x, u) \in Y: x(t)=\text { const. } u(t)=\text { const. a.e. }\}, \\
& C:=C\left(-r, \tau, \mathbb{R}^{n}\right) \times \mathscr{U}_{\mathrm{ad}}, \quad \tilde{C}:=C \cap \tilde{Y} .
\end{aligned}
$$

Identify $\mathbb{R}^{n} \times \mathbb{R}^{m}$ with $\tilde{Y}$ in the natural way. Then, (OPC) and (OSS) are equivalent to the corresponding problems $(\mathrm{P})$ and $(\tilde{\mathrm{P}})$, respectively. Defining the projection $\mathscr{P}: Y \rightarrow \tilde{Y}$ by

$$
\mathscr{P}(x, u):=\left((1 / \tau) \int_{0}^{\tau} x(s) d s,(1 / \tau) \int_{0}^{\tau} u(s) d s\right),
$$

one sees easily that conditions (i) and (ii) of Proposition 2.1 are satisfied. Concerning condition (iii) formulated in Remark 2.3, we start with a pair $\left(\lambda_{0}, z\right) \in \mathbb{R}_{+} \times \mathbb{R}^{n}$ satisfying the optimality condition for (OSS),

$$
\left[\lambda_{0} h^{\prime}\left(x^{0}, u^{0}\right)+z^{T} f^{\prime}\left(x^{0}, u^{0}\right)\right]\left(x, u-u^{0}\right) \geq 0,
$$

for all $x \in \mathbb{R}^{n}, u \in \Omega$. Define a continuous linear functional $z^{*}$ on $Z$ by

$$
z^{*}(\phi, \alpha)=\left(z^{T} / \tau\right)[\phi(\tau)+\alpha]
$$

for $(\phi, \alpha) \in Z=C\left(0, \tau ; \mathbb{R}^{n}\right) \times \mathbb{R}^{n}$. Then, one computes

$$
z^{*}\left(F^{\prime}\left(\bar{x}^{0}, \bar{u}^{0}\right)(x, u)\right)=z^{*}\left(F^{\prime}\left(\bar{x}^{0}, \bar{u}^{0}\right) \mathscr{P}(x, u)\right),
$$

for all $(x, u) \in Y$, thus establishing condition (iii). We conclude that also, in the case where $f_{x}\left(x^{0}, u^{0}\right)$ is a singular matrix, every pair $\left(x^{0}, u^{0}\right) \in \mathbb{R}^{n} \times \mathbb{R}^{m}$ satisfying the optimality condition (14) for (OSS) satisfies the optimality condition for (OPC). Observe that these arguments apparently do not go through for the general system (4). 
The results given above can be considered from a different point of view. As is well known (Ref. 8), Pontryagin's maximum principle allows one, in certain cases, to discern steady-state solutions which are merely optimal among steady states from those which are also optimal among periodic solutions. The maximum principle is based on strong variations around an optimal control $u^{0}(\cdot)$ having the form $(\hat{u} \in \Omega, \hat{t} \in[0, \tau))$,

$$
u_{\hat{t}, \hat{u}, h}(t)= \begin{cases}\hat{u}, & \text { for } t \in[t, t+h], \\ u^{0}(t), & \text { elsewhere. }\end{cases}
$$

Denoting the corresponding trajectories of (11) by $x\left(u_{\hat{t}, \hat{u}, h}\right)$, one sees that the corresponding limits

$$
\lim _{h \rightarrow 0}\left[x\left(u_{\hat{i}, \hat{u}, h}\right)-x\left(u^{0}\right)\right] / h
$$

are not Gateaux derivatives of $x$ with respect to $u$. Hence, the assumptions of Proposition 2.1 are not met.

\section{Conclusions}

We have proved an abstract result on nested optimization problems, with an application to optimal periodic control. It follows, in particular, that first-order necessary optimality conditions do not allow one to discern steady states which are merely optimal among steady states from those which are optimal among periodic solutions. This assertion, well known for ordinary differential equations, has been established for a class of neutral functional-differential equations. Alternatively, one might have tried to verify this result by analyzing the concrete form of the optimality conditions. However, this concrete form is unknown; in fact, its determination will require some nontrivial mathematical work taking into account the complicated duality theory of neutral functional differential equations (see Ref. 7 for the special case of autonomous equations). Hence, the approach taken here appears to be justified. Since the result of Section 2 is very general, it (or some variant of it) is likely to apply also to other classes of infinitedimensional systems.

The result of this paper (in some sense, negative) indicates that higherorder optimality conditions are likely to play an at least as important role for infinite-dimensional optimal periodic control problems as they do for finite-dimensional ones.

Furthermore, the result gives an explanation of why the Pontryagin maximum principle allows one to discern, in certain cases, optimal steadystate solutions which are merely optimal among steady states from those 
which are also optimal among periodic solutions. The maximum principle is based on strong variations, and the required differentiability assumption in Proposition 2.1 is not satisfied.

\section{Acknowledgment}

It is a pleasure to thank Prof. H. T. Banks for his invitation to work at Brown University.

\section{References}

1. Bittanti, S., Fronza, G., and Guarbadassi, G., Periodic Control: A Frequency Domain Approach, IEEE Transactions on Automatic Control, Vol. AC-18, pp. 33-38, 1973.

2. Bernstein, D. S., and Gilbert, E. G., Optimal Periodic Control: The II-Test Revisited, IEEE Transactions on Automatic Control, Vol. AC-25, pp. 673-684, 1980.

3. BeRnSTEIN, D. S., Control Constraints, Abnormality, and Improved Performance by Periodic Control, IEEE Transactions on Automatic Control, Vol. AC-30, pp. $367-378,1985$.

4. Colonius, F., Optimality for Periodic Control of Functional Differential Systems, Journal of Mathematical Analysis and Applications (to appear).

5. Lempio, F., and Zowe, J., Higher-Order Optimality Conditions, Modern Applied Mathematics, Edited by B. Korte, North-Holland Publishing Company, Amsterdam, Holland, pp. 147-193, 1982.

6. HALE, J., Theory of Functional Differential Equations, Springer-Verlag, Berlin, Germany, 1977.

7. SAlAmon, D., Observation and Control of Neutral Systems, Pitman, New York, New York, 1984.

8. GILBert, E. G., Optimal Periodic Control: A General Theory of Necessary Conditions, SIAM Journal on Control and Optimization, Vol. 15, pp. 717-746, 1977. 